\title{
Perspective
}

PERSPECTIVE Actualité en histoire de l'art

1 | 2009

Antiquité/Moyen Âge

\section{Les images médiévales à la recherche de nouveaux cadres}

Dominique Donadieu-Rigaut

\section{(2) OpenEdition}

1 Journals

Édition électronique

URL : http://journals.openedition.org/perspective/1896

DOI : 10.4000/perspective.1896

ISSN : 2269-7721

Éditeur

Institut national d'histoire de l'art

Édition imprimée

Date de publication : 31 mars 2009

Pagination : 146-151

ISSN : 1777-7852

\section{Référence électronique}

Dominique Donadieu-Rigaut, "Les images médiévales à la recherche de nouveaux cadres »,

Perspective [En ligne], 1 | 2009, mis en ligne le 21 juillet 2014, consulté le 01 octobre 2020. URL : http:// journals.openedition.org/perspective/1896; DOI : https://doi.org/10.4000/perspective.1896 


\section{Les images médiévales à la recherche de nouveaux cadres}

\section{Dominique Donadieu-Rigaut}

- Jérôme BASCHET, L'iconographie médiévale, (Folio Histoire inédit), Paris, Gallimard, 2008. 468 p., 48 fig. en coul. et en n. et b. ISBN : 978-2-07-034514-4; 9,90€.

- Brigitte D'HAINAUT-ZVENY, Les retables d'autel gothiques sculptés dans les anciens Pays-Bas. Raisons, formes et usages, (Classe des Beaux-Arts), Bruxelles, Académie royale de Belgique, 2008. 437 p., 78 fig. en coul. et en n. et b. ISBN : 978-2-8031-0248-8; $30 €$.

- Jean WIRTH, L'image à l'époque gothique (1140-1280), Paris, Cerf, 2008. 426 p., 143 fig. en coul. et en n. et b. ISBN : 978-2-204-07915-0; $42 €$.

Trois ouvrages parus en 2008 sur l'image médiévale semblent annoncer, chacun à sa manière, la fin de l'engouement pour le fractionnaire, le singulier et le discontinu qui a affecté les sciences humaines postmodernes. Loin d'adopter la posture de la " conclusion impossible " ou celle de la monographie n'engageant qu'elle-même, ces travaux cherchent à dégager de réels enjeux historiques et épistémologiques considérésà partird'échellestemporelles amples et de corpus documentaires étoffés. Dans L'iconographie médiévale, Jérôme Baschet rassemble, en un format éditorial accessible à tous, l'ensemble de ses réflexions méthodologiques sur l'image médiévale menées depuis une quinzaine d'années. La mise en livre d'articles jusqu'ici épars révèle la cohérence d'une pensée nuancée qui se méfie des théories et des systèmes trop bien suturés ne pouvant déboucher que sur des analyses mécanicistes et univoques. Jean Wirth poursuit, avec L'image à l'époque gothique, l'écriture de la trilogie ouverte par L'image à l'époque romane ${ }^{1}$ et bientôt close par L'image à la fin du Moyen Âge d'ores et déjà annoncée. La structure tripartite de cette vaste entreprise, ainsi que les titres des trois ouvrages, suffisent à nous convaincre qu'il s'agit pour l'auteur de se confronter à l'œuvre d'Émile Mâle pour mieux

1. Abbaye de Saint-Savinsur-Gartempe, voûte de la nef centrale, fin du $\mathrm{XI}^{\mathrm{e}}$ siècle. la revisiter. Enfin, Brigitte D'Hainaut-Zveny, avec Les retables d'autel gothique sculptés dans les anciens Pays-Bas, élabore autour de son objet d'étude - un corpus d'une centaine de retables - une démarche d'investigation personnelle se nourrissant sans a priori d'apports méthodologiques empruntés à diverses branches des sciences humaines qu'elle fait cohabiter pour le plus grand profit de sa problématique. Son travail se présente non comme une synthèse à la Michelet, mais comme un essai de " compréhension globale " du tableau d'autel.

Ces trois ouvrages intègrent les apports majeurs de ces dernières années à l'étude des images médiévales, notamment la reconnaissance de leur dimension foncièrement anthropologique. Les auteurs emploient d'ailleurs volontairement le terme d' " image ", et non celui d' " ouvre d'art " ou de "représentation", afin de faire leur le double héritage de Hans Belting ${ }^{2}$ et de Jean-Claude Schmitt $^{3}$ : les images ne peuvent être analysées en dehors de leurs usages et de leurs fonctions ${ }^{4}$, et ne peuvent se résumer aux images matérielles tant il est vrai qu'elles fonctionnent notamment en relation avecles représentations mentales surgissant lors des rêves ou dans la mémoire. En outre, la matérialité des œuvres doit être pleinement prise en considération puisqu'elle participe de leur effet : c'est l' " image-objet " de Baschet. Les trois auteurs reviennent également sur le phénomène central de l'incarnation dans la mesure où imago est un concept indispensable pour penser les rapports de ressemblance et de dissemblance entre Dieu et l'homme, mais aussi entre les trois personnes de la Trinité, le Fils étant, selon la célèbre formule d'Irénée de Lyon, la "visibilité du Père ". En ce sens, l'incarnation légitime non seulement la représentation anthropomorphique du divin mais aussi l'existence d'images porteuses d'une certaine forme de corporéité.

Cette volonté de saisir les objets médiévaux dans toute leur complexité et au-delà de ce qu'ils donnent à voir a poussé ces trois auteurs à repenser l'iconographie (que ce soit dans la lignée mâlienne ou panofskienne) à l'aune de l'anthropologie, de la théologie, voire de la sémiologie. Tous consacrent de belles pages soit à l'étude approfondie de telle ou telle œuvre (la voûte de Saint-Savin, entre autres, pour Baschet； fig. 1),

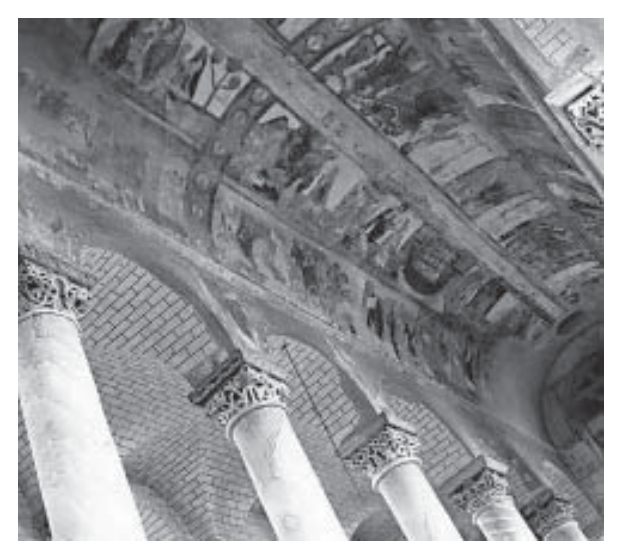


soit aux associations entre thèmes iconographiques (les figures des saints et la Passion du Christ pour D'Hainaut-Zveny) ou bien encore à des sujets particuliers (la roue de la Fortune ou le Jugement dernier pour Wirth).

\section{Comment articuler images et histoire ?}

Redéfinir l'iconographie passe nécessairement par une élucidation des rapports entre images et histoire. Aucun des trois auteurs n'est adepte de la Vie des formes ${ }^{5}$. Tous prennent en compte les relations étroites entre les œuvres et l'histoire, en faisant toutefois référence (de façon implicite ou explicite et à des degrés divers) à la notion de "pensée figurative » développéeparFrancastel ${ }^{6}, \mathrm{c}^{\prime}$ est-à-dire à la capacité qu'ont les images à produire du sens par elles-mêmes. Mais chacun situe à des niveaux différents les liens entre histoire et images. Pour Baschet, les images sont dans l'histoire dès lors qu'elles engagent des actes sociaux et contribuent à créer des interactions non seulement entre les hommes ici-bas, mais aussi entre les hommes et Dieu. En adoptant cette ligne très anthropologique, il rompt avec les conceptions de l'image comme simple "reflet de la réalité ", ou même comme "témoin des mentalités " d'une époque. En d'autres termes, il réfute leur caractère passif, préférant insister sur leur capacité opératoire à produire " du réel et de l'idéel ». D’Hainaut-Zveny, qui se réclame également d'une perspective anthropologique, a choisi d'intégrer à sa démarche la notion de culture proposée jadis par Pierre Bourdieu : " un univers symbolique où les symboles, plus ou moins partagés, servent à penser, à agir, à juger " ${ }^{7}$. Son objectif consiste à resituer les retables sculptés dans " un complexe d'idées et de sentiments " pour tenter de « voir les choses du point de vue de leurs utilisateurs" (D'HAINAUT-ZVENY, 2008, p. 20). Là encore, c'est par le biais de la fonction (ou, plus subtilement, des usages différenciés des retables) que l'auteur appréhende les images et leur donne sens dans l'histoire. Elle récuse tout principe de causalité historique au profit de la notion de coïncidence qui lui permet de construire un réseau signifiant de thématiques et de pratiques rituelles et/ou dévotionnelles autour de ses objets (fig. 2). Elle décèle ainsi la « cohérence organique » (p. 21) des retables dans les corrélations qu'ils entretiennent avec l'écheveau de la société environnante.
Les relations établies par Wirth entre histoire et images s'avèrent plus problématiques dans la mesure où il tire

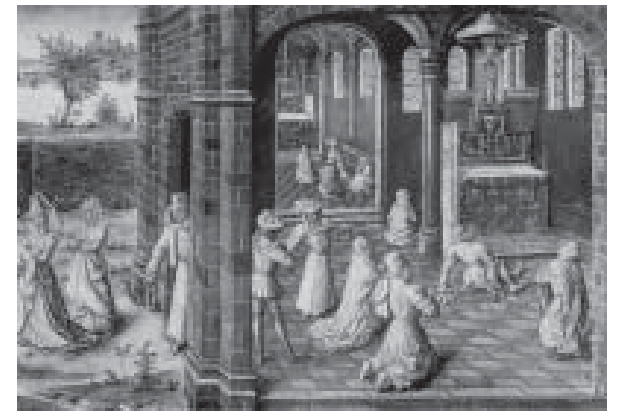
la définition de l'image gothique du côté de la sémiologie. En débutant son ouvrage par un savant chapitre sur les réflexions scolastiques ayant érigé l'image en signe, il semble sous-entendre que la théologie est première, ou du moins que le chercheur a nécessairement besoin de s'appuyer sur la scolastique pour penser les images médiévales. La prééminence qu'il accorde aux principes théologiques est tout entière au service du plan général de son ouvrage visant à prouver qu'il y a rupture entre une époque romane qui serait entièrement façonnée par l'idéologie monastique du mépris du monde, et une époque gothique plus ouverte au monde profane, réconciliée avec la chair et les sens.

Les rapports entre images et histoire engagent également la question des transformations des œuvres dans le temps. Wirth, renouant avec des conceptions historiographiques somme toute assez évolutionnistes (pour lui, l'époque gothique est " une véritable époque de progrès global, à la fois technique, politique et social ", p. 388) n'en est pas moins très attentif à une datation précise qui l'amène à nuancer son discours. Il décèle notamment des décalages chronologiques entre la pensée scolastique et les "innovations " iconiques. Quelques-uns des sourires apparaissant sur les lèvres des Élus (porte du Prince de la cathédrale de Bamberg; fig. 3), des anges (façade occidentale de la cathédrale de Reims) ou encore de la Vierge (portail Saint-Honoré de la cathédrale

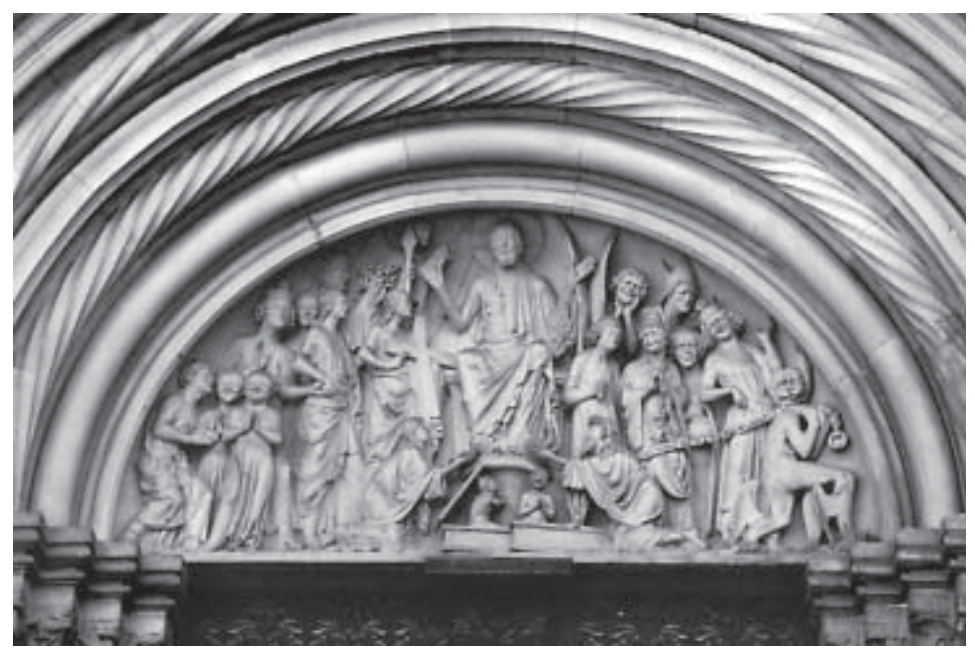

2. Audenarde, Jean Le Tavernier, Les miracles de Notre-Dame, $2^{\mathrm{e}}$ partie, vers 1458-1460: La Vierge guérissant un estropié, fol. 58, Paris, Bibliothèque nationale de France [D'HAINAUTZVENY, 2008, couverture].

3. Cathédrale

de Bamberg, tympan de la porte du Prince, Jugement dernier, vers 1225 . 


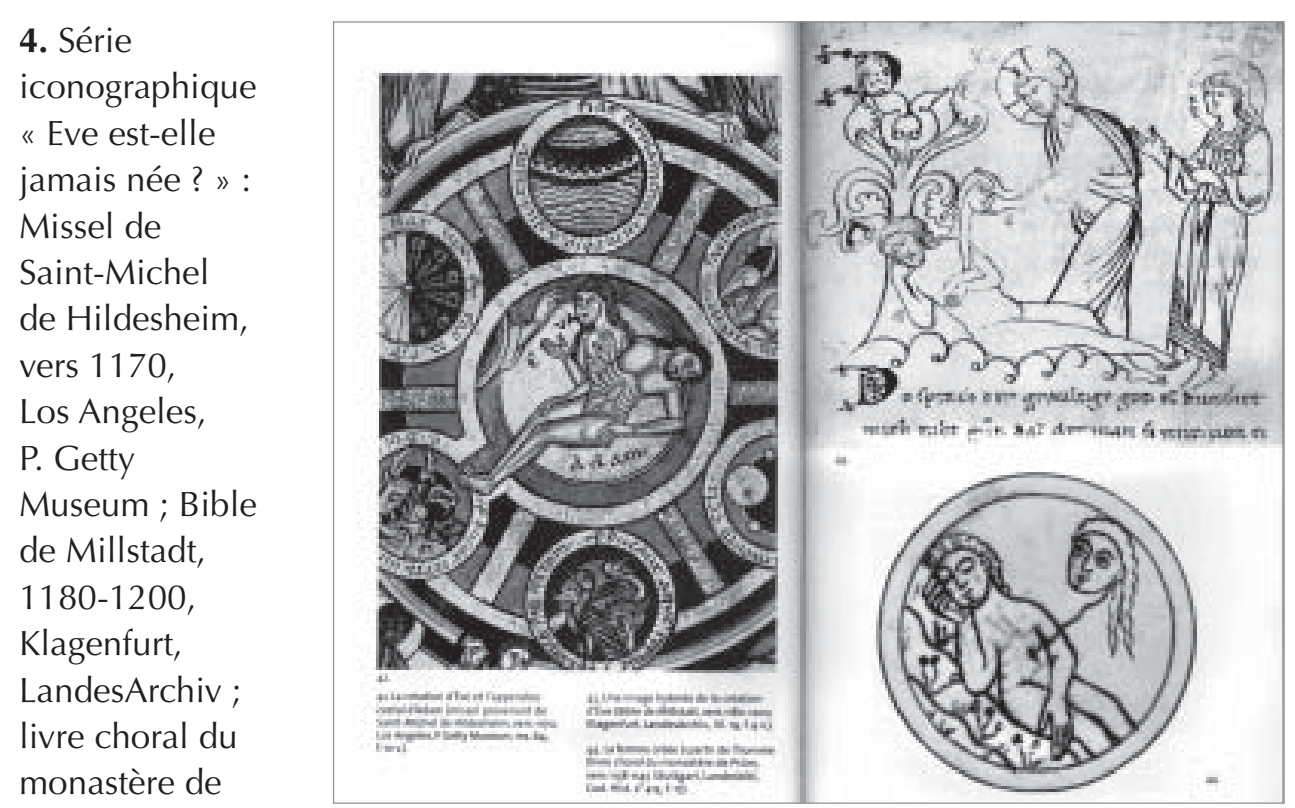

Prüm, 1138-

1147, Stuttgart,

Landesbibliothek

[BASCHET, 2008,

fig. 42-44]

5. Attribué

à Vrancke

vander Stockt,

Adoration du

Saint-Sacrement,

vers 1500

Anvers,

Koninklijk

Museum voor

schone Kunsten.
d'Amiens) précèdent les théories hylémorphistes telles qu'elles furent réactualisées par Albert le Grand et Thomas d'Aquin. La grande statuaire semble donc avoir formulé avant les théologiens l'idée que le corps est le miroir de l'âme.

Baschet, en revanche, réfute avec force la notiond'unitéhistoriquequipermettraitd'englober la totalité des ouvres d'une période donnée en un même ensemble homogène. Il insiste au contraire sur l'inventivité foncière et permanente des images médiévales, une inventivité niée par l'iconographie traditionnelle dont l'un des objectifs est de définir les conventions de l'art religieux, ses modèles et ses stéréotypes. C'est précisément la raison pour laquelle l'auteur a mis au point une méthode d' "iconographie sérielle ", qui vise à éclairer la façon dont les images s'apparentent les unes aux autres et font sens dans cette relativité dynamique (fig. 4). La construction de corpus sériels par le chercheur s'avère alors cruciale pour l'analyse non seulement des images ellesmêmes mais aussi de ce qui se joue entre les images, dans les citations, les emprunts, les glissements et les écarts. Cette approche implique des échelles temporelles pluriséculaires permettant de percevoir les phénomènes de tâtonnement, de coexistence temporaire ou d'effacement progressif de certains thèmes iconographiques au profit d'autres, comme Baschet en a déjà fait la démonstration dans un ouvrage précédant, à propos du sein d'Abraham ${ }^{8}$.

La "longue durée» est également l'un des soucis méthodologiques D'Hainaut-Zveny. Bien qu'elle focalise son attention sur les retables des anciens Pays-Bas à la fin du Moyen Âge, l'auteur prend soin de mettre en perspective son objet d'étude, y compris d'un point de vue historiographique. C'est ainsi qu'elle repère trois moments distincts et successifs dans l'histoire des retables, sans pour autant nier les phénomènes de coexistence : "le temps des reliques ", "le temps des images", "le temps du Saint-Sacrement" (fig. 5). Son argumentation vise à montrer qu'entre le XIV ${ }^{\mathrm{e}}$ siècle et le milieu du XVI ${ }^{\mathrm{e}}$ siècle, les images des retables ont assumé seules le rôle de présentification ${ }^{9}$ du sacré sur l'autel, sans que les reliques ou le Saint-Sacrement ne soient nécessairement intégrés à la structure de ces "images-objets". Pour elle, le moteur essentiel de ces mutations réside dans les relations variables que les fidèles et le clergé entretiennent avec la présence divine ici-bas. Les rapports à la fois complémentaires et concurrentiels entre images, reliques et hostie consacrée semblent ainsi présider à cette histoire complexe des retables sculptés.

\section{Les images et leurs " lieux"}

Si la conjonction des images et du temps historique retient les trois auteurs, Baschet et D'HainautZveny consacrent à la question cruciale de l'espace, ou plutôt des lieux de l'image ${ }^{10}$, des pages essentielles. Depuis les travaux novateurs de Paul Zumthor ${ }^{11}$, nous savons que le Moyen Âge ignore l'espace unifié, homogène et continu en tant que catégorie abstraite, indépendante des objets qui l'habitent. C'est en termes de réseaux multipolaires que la question se pose, chaque locus étant le siège d'une présence, d'une force, d'un sentiment d'appartenance à partir duquel les rapports sociaux s'organisent, les entités sociales se constituent. Il est donc impropre et anachronique de parler d'espace tel que nous l'entendons aujourd'hui pour la période médiévale, aussi bien en ce qui concerne la structuration de la chrétienté que

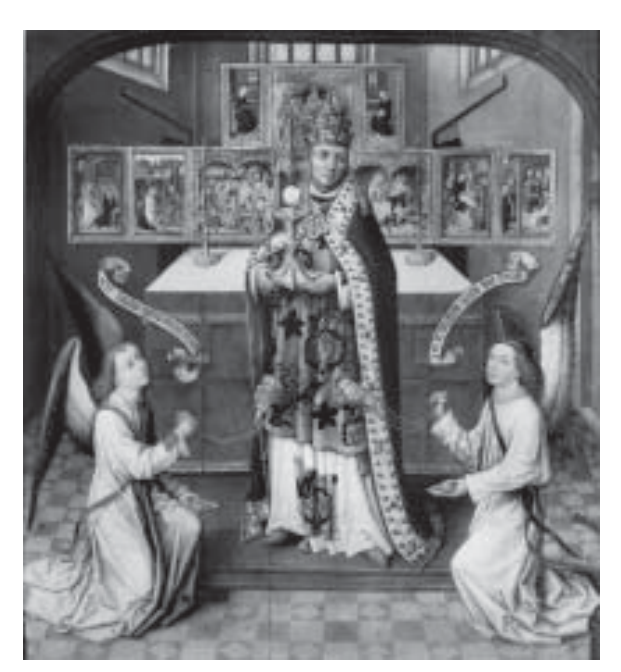
la composition d'une image matérielle $^{12}$. Jérôme Baschet souligne d'ailleurs à quel point on ne saurait isoler les lieux de l'image des lieux sociaux tant il est vrai que les images- 
objets ont grandement contribué à la polarisation de la société féodale. Il focalise son attention sur le bâtiment ecclésial, centre d'un ensemble de cercles concentriques allant du cimetière à la chrétienté tout entière en passant par les échelles intermédiaires que sont la paroisse et le diocèse. En effet, l'église, ancrée dans un territoire, incarne non seulement le creuset où se réalise l'unité de l'Ecclesia (par le biais des sacrements), mais aussi le lieu par excellence des images puisque les ornamenta et les imagines contribuent à la magnifier, c'est-à-dire à la désigner comme sacrée. Abandonnant la dichotomie traditionnelle sacré/ profane, l'auteur propose de penser ces configurations réelles et idéelles en termes "d'enveloppes sacrales ". Dès lors, il met en évidence non plus une frontière symbolique exclusive mais des successions de seuils menant à des degrés de sacralité marqués précisément par la présence d'images. Ainsi, le décor ecclésial, compris dans sa complexité et ses interactions (peintures murales, sculptures internes et externes, retables, luminaires et objets liturgiques), manifeste tout à la fois les zones de polarisation de l'édifice (autels consacrés), ses multiples divisions internes (mur diaphragme, jubé...), et son axialité dynamique et orientée (locus/iter).

L'importance des liens entre liturgie, espace ecclésial, organisation sociale et images est également soulignée par D’Hainaut-Zveny. Pour elle, les retables ordonnent l'agencement interne du bâtiment dans la mesure où ils contribuent à en faire un espace partagé clercs/laïcs. Par leur fonction ambivalente, ces tableaux d'autels sculptés renforcent d'une part le pouvoir sacerdotal en intensifiant les gestes et les paroles du prêtre lors du rituel eucharistique, et favorisent d'autre part les pratiques dévotionnelles en créant les conditions d'une relation à Dieu personnalisée. Le retable règle donc les rythmes de la vie religieuse et les modalités d'occupation des lieux en faisant cohabiter le temps de la messe et le temps des dévotions, personnelles ou collectives (familles, confréries, corporations...).

Si les retables sculptés sont dans l'église et sur l'autel, ils possèdent eux-mêmes leur propre configuration en volume. D'Hainaut-Zveny montre que chacune des multiples alvéoles de ces architectures en bois correspond à un petit théâtre de mémoire où vient se lover le rapport projectif du dévot au mystère divin. La disposition en cercle des personnages peuplant ces cavités ménage une place vide en amont de la scène : celle du fidèle, invité à faire sa propre expérience de la présence divine en concentrant dans ces écrins dorés les mouvements de son âme. Ainsi, la juxtaposition de niches permet l'absorption du sujet chrétien dans les lieux de l'Histoire Sainte. L'auteur oppose à cet égard les polyptyques des Pays-Bas aux retables peints en Italie à la même époque : là où le système perspectif institue la représentation en créant une distance entre celui qui regarde et le représenté, les " concrétisations imaginaires " des Pays-Bas refusent l'objectivation de la représentation, favorisant ainsi l'investissement du fidèle dans un dispositif en relief.

Les trois ouvrages montrent combien les images font les lieux et comment les lieux s'emboîtent ; les images réagissent les unes par rapport aux autres et suscitent dans la mémoire d'autres images, matériellement absentes ; les images prennent sens en interaction avec les gestes, les pensées, les affects des hommes. C'est ce que Baschet propose d'appeler "l'iconographie relationnelle " : les sens possibles d'une œuvre ne résident pas uniquement dans ses configurations plastiques et ses thématiques; les nouds de relations internes au discours figuratif interagissent avec les situations sociales qui se jouent devant les œuvres, notamment dans l'église.

\section{Le visible, l'exhibé, le dissimulé}

On peut difficilement parler d'images sans se soucier de la question du regard ${ }^{13}$. Non seulement parce que les hommes du Moyen Âge regardaient (ou non) les images-objets, mais aussi parce que celles-ci fixaient parfois les hommes de leurs yeux perçants. En outre, les représentations ne sont pas totalement étrangères aux différentes conceptions scientifiques de la vision, ni même à ce que nous considérons comme visible ou invisible, représentable ou non représentable, beau ou laid.

Wirth puise dans le Roman de la rose (12301270) matière à relier la vision, le reflet, la beauté et le désir. Il décèle dans ce grand texte de la littérature courtoise une légitimation du savoir passant par les sens et dont la nature serait l'objet principal. La totalité du monde étant 
alors considérée comme " un livre écrit du doigt de Dieu ", l'image acquiert son sens spirituel dès lors qu'elle cherche à reproduire avec ressemblance l'aspect de ce monde tangible, scruté dans ses moindres détails. Le beau artistique n'a donc pas de réalité en soi : il n'est que l'émanation du beau naturel, reproduit dans ses proportions et ses qualités sensibles, voire sensuelles. Cette mutation culturelle constitue pour l'auteur la clé d'un art gothique résolument optimiste et anti-ascétique, ayant émergé dès le milieu du XII siècle dans le reflux de la réforme grégorienne. Loin de lui, cependant, l'idée simpliste d'une époque gothique qui se dégagerait de l'emprise de l'Église pour représenter un monde ici-bas résolument profane. Le nouveau regard que le sujet chrétien pose sur la nature participe de la divination de la chair christique dans le cadre eucharistique. L'heure n'est plus au spirituel invisible. Le vieil adage "heureux ceux qui croient sans avoir vu " (Jean 20,29), qui permettait de taxer d'incrédulité le geste de saint Thomas, cède la place à l'adoration de la divinité incarnée, visible, représentable, palpable et consommable. Wirth propose donc de substituer à la césure visible/invisible, le binôme naturel/surnaturel, la transsubstantiation étant la manifestation la plus accomplie du surnaturel sur terre.

À l'inverse, Baschet vide en quelque sorte l'ici-bas de sa consistance afin de mieux combattre la notion $d^{\prime}$ " image-miroir ». À partir d'exemples touchant à des actes rituels, il démontre que les images matérielles ne peuvent en aucun cas refléter le réel dans la mesure où, pour la pensée figurale du Moyen Âge, ce sont les réalités d'ici-bas qui constituent un pâle reflet des vérités célestes, par nature invisibles. Le chapiteau montrant le Christ lavant les pieds des apôtres dans le cloître de Moissac ne doit donc pas être analysé comme la simple représentation in situ de la cérémonie du mandatum impliquant l'abbé et ses moines. Ce serait plutôt le rite monastique qui reflèterait, au sein du monde claustral, la vérité christique. L'image, elle, serait là pour remémorer et manifester le prototype divin, c'est-à-dire donner sens aux gestes des moines s'effectuant dans le temps historique. Ainsi, la sculpture n'illustre rien : elle dévoile les raisons de l'efficacité rituelle, tout en métamorphosant temporairement l'abbé en figura du Christ.
Et en dehors du déroulement du mandatum, l'image constitue la trace durable du rituel éphémère.

D’Hainaut-Zveny, étant donné la spécificité de ses objets d'étude, place au cœur de sa réflexion sur le visible et l'invisible la question du " comment rendre présent le sacré ". Elle analyse l'apparition, au XIV siècle, des volets mobiles sur les retables comme l'émergence d'un dispositif permettant de réguler l'accès à la présence divine. Cette " innovation " n'est pas à envisager comme une simple modification formelle de la structure des retables, ni même comme une adaptation "mécanique " du tableau d'autel aux pratiques liturgiques. En orchestrant une alternance entre ostentation et occultation, les volets transforment le statut même des images. L'auteur en fait la démonstration en s'appuyant sur la définition du sacré selon Émile Durkheim comme «ce qui doit être tenu à distance ", " ce qui doit être caché ${ }^{14}$. Les images sculptées des huches, dissimulées par les images peintes des volets, acquièrent un degré de sacralisation inégalé, précisément parce qu'elles sont le siège d'une privation visuelle. Ainsi, les volets sacralisent les images, où plutôt leur confèrent cette capacité à rendre présent le sacré dès lors qu'ils contraignent la vision à l'incomplétude.

L'accessibilité ou la non-accessibilité constitue donc l'un des paramètres à prendre en considération dans l'analyse des images médiévales. Faut-il rappeler que nombre de sculptures ou de vitraux, traités avec un soin extrême, ont été placés sciemment hors de portée de l'œil humain, comme s'ils étaient dévolus d'abord et avant tout à l'œil omniprésent et omnipotent de Dieu ? La relation entre le regard humain et l'image n'est donc pas, au Moyen Âge, une relation absolument nécessaire. Pour certaines images, " être là " s'avère au moins aussi important qu'être vues.

Ces trois livres, d'une densité remarquable (et qui recèlent encore bien d'autres champs non évoqués ici) méritent d'être lus et relus afin d'y puiser matière à réflexion, matière à expérimentation méthodologique, en infirmant ou confirmant les hypothèses et les démarches proposées. Leur volonté de se positionner vis-à-vis des " grands héritages " en fait des outils indispensables pour tous ceux qui souhaitent entreprendre ou poursuivre un vrai travail de questionnement sur l'image médiévale. 
1. Jean Wirth, L'image à l'époque romane, Paris, 1999.

2. Hans Belting, Image et culte. Une histoire de l'image avant l'époque de l'art, Paris, 1998 [éd. orig. : Bild und Kult. Eine Geschichte des Bildes vor dem Zeitalter der Kunst, Munich, 1990].

3. Jean-Claude Schmitt, Le corps des images. Essai sur la culture visuelle au Moyen Âge, Paris, 2002.

4. Jérôme Baschet, Jean-Claude Schmitt éd., L'image fonctions et usages des images dans l'Occident médiéval, (Cahiers du Léopard d'Or, 5), Paris, 1996.

5. Henri Focillon, Vie des formes, Paris, 1934 ; Pierre Wat éd. Henri Focillon, Paris, 2007.

6. Pierre Francastel, La figure et le lieu, l'ordre visuel du Quattrocento, Paris, 1967, en particulier p. 9-21.

7. Pierre Bourdieu, Roger Chartier, Robert Darnton, " Dialogue à propos de l'histoire culturelle ", dans Actes de la recherche en sciences sociales, 59, 1985, p. 87.

8. Jérôme Baschet, Le sein du Père. Abraham et la paternité dans l'Occident médiéval, Paris, 2000.

9. Nous devons cette notion à Jean-Pierre Vernant qui l'emploie dans son ouvrage Mythe et pensée chez les Grecs, Paris, 1996 ( "De la présentification de l'invisible à l'imitation de l'apparence ").

10. Jérôme Baschet, Lieu sacré, lieu d'images. Les fresques de Bominaco (Abbruzes, 1263): thèmes, parcours, fonctions, Paris/Rome, 1991.

11. Paul Zumthor, La mesure du monde, représentation de l'espace au Moyen Âge, Paris, 1993, en particulier chap. 3, "Lieux et non-lieux".

12. Jean-Claude Schmitt, « De l'espace aux lieux : les images médiévales ", dans Construction de l'espace au Moyen Âge. Pratiques et représentations, (colloque, Mulhouse, 2006), Paris, 2007, p. 317-346.

13. Herbert Kessler, Seeing Medieval Art, Peterborough, 2004.

14. Émile Durkheim, Les formes élémentaires de la vie religieuse, Paris, 1960, p. 431-432.

Dominique Donadieu-Rigaut, EHESS

dom.donadieu@wanadoo.fr

\section{Le Trecento en Italie, entre Toscane, Lombardie et Vénétie}

\section{Serena Romano}

- L'eredità di Giotto: arte a Firenze 1340-1375, Angelo Tartuferi éd., (cat. expo., Florence, Galleria degli Uffizi, 2008), Florence, Giunti, 2008. 256 p., fig. en n. et b. et en coul. ISBN : 978-8-8090-6031-9; $34 €$.

- Fantasie und Handwerk: Cennino Cennini und die Tradition der toskanischen Malerei von Giotto bis Lorenzo Monaco, WolfDietrich Löhr, Stefan Weppelmann éd., (cat. expo., Berlin, Gemäldegalerie, Staatliche Museen zu Berlin), Munich, Hirmer, 2008. 336 p., 213 fig. en n. et b. et 64 en coul., ISBN : 978-3-7774-4115-3;39,90€.

- Giovanni da Milano: capolavori del gotico fra Lombardia e Toscana, Daniela Parenti éd., (cat. expo., Florence, Galleria dell'Accademia, 2008), Florence, Giunti, 2008. 320 p., fig. en n. et b. et en coul. ISBN : 978-8-8090-5978-8; $45 €$.

L'été 2008, à Florence, fut un été trecentesque avec deux expositions jumelles, et même siamoises, puisqu'elles étaient partiellement entrelacées : Giovanni da Milano à la Galleria dell'Accademia et L'eredità di Giotto aux Offices, respectivement sous la direction de Daniela Parenti et d'Angelo Tartuferi, furent à la fois un événement et une fête pour les yeux, et une extraordinaire occasion d'étude pour les spécialistes.

Il convient de suivre l'ordre chronologique en commençant par L'eredità di Giotto, qui posait le problème le plus précoce, celui de l'atelier, ou plutôt des ateliers du maître à partir des années 1310, une période qui s'ouvre avec le polyptyque de Raleigh $\left(n^{\circ} 1\right)$, le prêt le plus important de l'exposition, autrefois retable de la chapelle Peruzzi de Santa Croce. Cette peinture spectaculaire, datée de 1310-1315 environ, a attiré en effet l'attention sur la seconde décennie du Trecento, décennie clé pendant laquelle Giotto se fixe à Florence et intensifie les achats de terrains et de maisons dans son Mugello natal ${ }^{1}$. Solidement reliée aux grands banquiers, tels que les Peruzzi, et aux ordres religieux, dont les franciscains, qui avaient soutenu le peintre dès ses travaux à Assise, à Rimini et à Padoue, l'activité de Giotto continue à s'épanouir. La restauration du Crucifix d'Ognissanti, que Marco Ciatti dirige à l'Opificio delle Pietre Dure, contribuera 